Joanna JERECZEK-LIPINSKA

Université de Gdańsk

\title{
DE LA DYNAMIQUE DANS L'EVOLUTION DU DISCOURS POLITIQUE
}

Cette recherche s'inscrit d'un côté dans l'analyse discursive du discours politique et de l'autre dans la logométrie et le traitement assisté par ordinateur de ce même discours. Elle essaie de constater l'impact qu'a eu, qu'a et que peut encore avoir l'avènement de nouveaux dispositifs électroniques dans la communication politique. L'objet donc n'est pas tant de retracer les manifestations aussi diverses et variées du discours politique sur Internet, mais plutôt d'analyser les évolutions en cours dans le discours politique dans sa version traditionnelle. Ce qui nous intéresse ici, ce sont les évolutions qui sont dues au média nouveau et à ce combat politique qui, désormais, se joue également sur la scène politique virtuelle.

Dans cette perspective, cet article s'inscrit d'un côté dans la lignée des travaux axés sur le discours politique - P. Charaudeau (2005), C. SAlaVASTRU (2004), K. Kerbrat-OrECCHIONi (1998), J. MOUCHON (1997), de l'autre, essaie de confronter les hypothèses avec les résultats des analyses qui ont été déjà menées par D. MAYAFFRE (2004) et qui portaient sur le discours présidentiel sous la $\mathrm{V}^{\mathrm{e}}$ République ou d'E. RAULET (2004) qui avait analysé les discours de candidature des présidentiables à l'élection présidentielle en 2002.

Cette recherche s'effectue à partir du corpus recueilli sur Internet dans la période allant du mois de janvier 2005 au mois de novembre 2006 et il englobe les interventions des deux présidentiables représentant deux partis adverses, à savoir Ségolène Royal (Parti Socialiste) et Nicolas Sarkozy (UMP- Union pour un Mouvement Populaire).

Nous nous proposons d'analyser et de cerner la dynamique dans laquelle s'est engagée cette forme d'expression sans pour autant en tirer les conclusions définitives tout en sachant que l'évolution est en cours et qu'elle continue à façonner ce langage.

\section{Diverses formes de la discursivité politique}

Le pouvoir s'exerce, mais surtout s'acquiert par les mots. A l'époque de la communication politique, chaque homme politique a à sa disposition tout un éventail de dispositifs. D'un côté, l'interview à la télévision, à la radio ou dans la presse écrite, de l'autre, les déplacements et les meetings politiques. Viennent ensuite le slogan publicitaire faisant partie du marketing politique coordonné par les conseillers en communication ainsi que le livre politique. Dans ce cadre là justement, Nicolas Sarkozy a fait couler beaucoup d'encre ${ }^{1}$ et il est également

\footnotetext{
${ }^{1}$ Voici quelques titres parus en 2006 :

Jean Jacques ReBOUX (2006), Lettre ouverte à Nicolas Sarkozy, ministre des libertés policières. Candidat à la Présidence de la République, Après la Lune.

Philippe Cohen, Richard Malka (2006), La face karchée de Sarkozy, Vents d'Ouest.

David D' EQuainville (2006), Présidentielles : Sarkozy. 1997-2006, Nicolas a dit, Anabet.

Pascale Nivelle, Elise Karlin (2006), Les Sarkozy. Une famille française, Calmann-Levy.

Frederic Charpier (2006), Nicolas Sarkozy. Enquête sur un homme de pouvoir, Presses de la Cité.
} 
l'auteur de livres ${ }^{2}$. De même, Ségolène Royal, est le sujet de plusieurs ouvrages parus récemment ${ }^{3}$ et elle est en train de coécrire, sur son blog, un livre en ligne «Désir d'avenir». Le livre politique a, en France, une importance à ne pas négliger, l'illustration en est le fait que la sortie récente du livre de Lionel Jospin ${ }^{4}$ a fait croire à beaucoup, qu'il serait candidat, et que son livre n'est qu'un programme de campagne. Ces informations ne se sont pas confirmées par la suite.

Le dernier dispositif est celui qui nous intéresse le plus ici, à savoir, le site Web, forum ou mieux encore, le blog. Chacun de ces dispositifs, avec sa spécificité, contribue à aider le politique dans sa lutte pour l'obtention du pouvoir (SALAVASTRU, 2004: 175). Le blog, étant une sorte de cahier de bord d'un politique, est censé fournir au public, sa lecture d'actualité. Nous constatons que le blog est un média nouveau qui a déjà laissé des traces durables sur le discours politique. Tout média nouveau adopte le langage des médias existant pour se forger ensuite son propre mode d'expression et ensuite influencer les formes d'expression en place. Tel a été le cas du blog qui entraîne le discours politique dans une dynamique évolutive sans précédent.

Comme nous venons de le démontrer, le message que transmet le politique peut atteindre le public par l'intermédiaire de plusieurs formes de la discursivité, et en passant par différents canaux de communication, avec, à chaque fois, quelques spécificités dues au courroie de transmission. Le but est d'atteindre ce que $\mathrm{C}$. SALAVASTRU (2004:30) appelle «la performance discursive » maximale. Or, de plus en plus souvent, toutes les formes de la discursivité politique des présidentiables, sont ensuite transférées sur leurs blogs. La cohérence intradiscursive, mais surtout interdiscursive oblige. En effet, le politique ne peut plus vraiment adapter son discours en fonction du dispositif car tout ressortirait sur Internet et surtout mettrait en question sa fiabilité. Ainsi, le politique a dû, emprunter au cyberlangage politique, et s'en servir partout, y compris, en dehors d'Internet. Car ce qui est encore possible pour un politique moyen ${ }^{5}$, ne convient pas vraiment au présidentiable, à qui on pourrait reprocher de tenir un double discours.

L'objet est ici de démontrer l'impact des médias électroniques sur la parole politique ailleurs que sur internet. Pour ce faire, nous avons sélectionné deux politiques considérés comme novateurs dans leurs discours et nous retracerons cette évolution en démontrant qu'elle s'opère en dehors du clivage politique droite/gauche. L'évolution qu'a enclenchée l'avènement du blog, affecte tout aussi les acteurs du domaine politique que ceux de tous les autres médias. L'instance politique peut désormais toucher directement sa cible sans passer par le filtre des

\footnotetext{
${ }^{2}$ Nicolas SARKOZY (2006), Témoignage, XO (EDITIONS).

Nicolas SARKOZY, Thibaud COLLIN, Philippe VERDIN (2005), La République, les religions, l'espérance, Pocket.

${ }^{3}$ Nathalie Guiot (2006), Présidentielles : Royal. 1990-2006, Ségolène a dit..., Anabet.

Aymeric Mantoux, Benoist Simmat (2006), Ségolène Royal. La dame aux deux visages, L'archipel. Laurent PFAADT (2006), Ségolène Royal décryptée de A à Z, City.

Marie-Eve Malouines, Carl Meeus (2006), La madone et le culbuto. Ou l'inlassable ambition de Ségolène Royal et François Hollande, Fayard.

${ }^{4}$ L. JosPIN (2005), Le monde comme je le vois, Editions Gallimard.

${ }^{5}$ Julien Dray tient son blog sur Skyblog où il emploie un parler jeune tout en occupant sa fonction de porte-parole au sein du Parti Socialiste avec donc ses interventions plus modérées au nom du Parti.
} 
journalistes. Désormais, ce journaliste, face à la communication en direct qui se fait entre le politique et le citoyen, est devenu l'évaluateur, il évalue les propos et les actes politiques, il distribue les points, il traque les lapsus, les gaffes et les mensonges. En d'autres termes, il surveille l'univers politique. Ainsi, les médias se constituent progressivement en autorité et deviennent une sorte de «gardiens de promesses »(GARAPAN, 1996).

Il s'ensuit que le discours politique prend une relative indépendance et acquiert de nouvelles fonctions. En effet, Internet permet au politique d'informer son public, voire, d'assurer le socle informatif, de le mobiliser, de l'aider enfin dans sa prise de décision. Mais là, où cet outil est le plus précieux, pertinent et original, est justement l'espace qu'il offre, pour la discussion directe (descendante et ascendante) et pour le débat, l'interaction et l'échange. C'est cette nouvelle dimension qui a, à ce point, affecté le discours politique. Car, tout débat nécessite un certain nombre de conditions dont la compréhension des enjeux ainsi que l'implication du public dans ceux-ci, sont les plus importants. Ainsi, pour qu'un public veuille intervenir dans un débat, il doit d'abord en comprendre le sens, ensuite se sentir concerné et percevoir l'intérêt, voire l'impact direct de son intervention.

Ces trois conditions sont indispensables pour un débat démocratique, et c'est dans cette optique qu'apparaissent les trois mots d'ordre qui marquent l'univers politique d'aujourd'hui, à savoir, la proximité, la transparence et l'affect. Car, le public devrait pouvoir comprendre les enjeux, d'où le souci d'explication, tout comme il devrait pouvoir se sentir concerné, d'où le concept de proximité et enfin, il devrait mesurer l'intérêt de son effort participatif.

Quel sont donc ces traits caractéristiques évolutifs des discours de Nicolas Sarkozy et de Ségolène Royal et qui font qu'ils sont à opposer, respectivement, non pas à Lionel Jospin, à François Mitterand ou à Charles de Gaulle, mais à Dominique Strauss-Kahn, Laurent Fabius pour S. Royal et Jacques Chirac, Dominique de Villepin ou Michèle Alliot-Marie pour N. Sarkozy.

\section{Le descriptif du corpus et la méthodologie de la recherche}

Pour démontrer la constante évolutive qui va au-delà du clivage gauche-droite, nous avons étudié les discours d'un représentant de la droite - Nicolas Sarkozy (UMP) et d'une représentante de la gauche - Ségolène Royal (PS). Notre sélection a été motivée par le simple fait que l'un et l'autre sont considérés comme novateurs en matière de communication politique au sein de leurs familles politiques. Ainsi, 33 discours de N.S. et 29 de S.R, toutes formes de la discursivité confondues, ont été soumis à quelques tests dont l'analyse discursive et l'analyse logométrique. Ce sont donc essentiellement les discours prononcés lors des meetings, des discussions au sein des partis, des interventions sur leurs blogs et des interviews sur les plateaux de télévision, à la radio et enfin des entretiens parus dans la presse écrite. Toutes ces interventions ayant ensuite été transférées sur le blog, et ceci, soit dans la version tapée, soit sous forme de podcast vidéo. Nous avons, à chaque fois, confronté l'original avec ce qui est paru sur le blog pour confirmer qu'il s'agit bien d'une version intacte, sans aucune modification, sans aucune coupure, sans aucun raccourci.

Quant à l'analyse discursive, ont été pris en compte le contexte de l'énonciation, les caractéristiques des locuteurs ainsi que les caractéristiques 
sémantiques de l'énoncé ainsi que les productions et réceptions de ces discours. Cette analyse discursive a été ensuite affinée et concrétisée par les études de type statistique dont les résultats ont été confrontés à ceux obtenus par Damon MAYAFFRE et impliquant les Présidents sous la $\mathrm{V}^{\mathrm{e}}$ République. Le corpus analysé est comparatif et contrastif (N. Sarkozy vs S. Royal) et diachronique. Il englobe tous les discours transférés sur le blog entre 2005 et 2006 dans la période préélectorale soit une soixantaine de discours. Nous écartons de cette présentation, tout jugement quant à la validité des arguments présentés.

Trois problématiques socio-linguistiques ont été formulées dés l'abord de cette recherche. Premièrement, y a-t-il une spécificité linguistique sarkozyste et une spécificité royaliste? Deuxièmement, ces deux politiques ont-ils des points communs dans leur discours ? Troisièmement, pourquoi et comment apparaissentils en rupture, non pas l'un par rapport à l'autre, mais face à ses rivaux au sein de leurs partis respectifs.

La réponse à la première problématique semble s'imposer. En effet, on peut parler de ce que D. MAYAFFRE (2004) qualifie d" «identité discursive », due au fait que nous avons affaire ici à deux personnalités différentes, avec deux statuts différents et donc deux discours. Nicolas Sarkozy est Ministre de l'Intérieur au gouvernement de D. de Villepin alors que Ségolène Royal est Présidente de conseil régional en Poitou-Charentes. Mais surtout leur appartenance politique fait qu'ils utilisent un discours différent. Pourtant, ces différences semblent, ces derniers temps, s'estomper et le clivage droite-gauche n'est plus tellement visible dans le discours, comme il ne l'est plus vraiment ni dans les idées et ni dans les projets.

Nous allons illustrer d'un côté ce facteur individuel, qui existe certes, de l'autre, ce qui fait que les deux discours tendent à se rapprocher.

\section{Le discours politique évolutif}

MAYAFFRE (2004 : 24) constate que « les présidents français prononcent tous des discours stéréotypés, politiquement policés, idéologiquement modérés. » Qu'en est-il aujourd'hui ?

Dans cette perspective, observons l'évolution sémantique et syntaxique du discours des présidentiables.

\subsection{La guerre des mots - du lexème au vocable}

Les représentants et les militants de chaque parti se forgent un lexique qui lui est propre. Mais, aujourd'hui la tendance est de s'emparer des mots associés jusque-là aux adversaires pour les teinter des valeurs péjoratives. Tel était le cas du qualificatif libéral, qui, plongé systématiquement (par exemple durant la campagne portant sur le Traité de Constitution) dans un contexte très négatif, a fini par perdre ses valeurs neutres (libéral relatif au membre d'un parti libéral) et même positives (relatives à toutes les libertés). Aujourd'hui en politique, tout le monde en convient, la notion libéral est devenue tout un argument à part, voire même une insulte. C'est même un argument incontournable et, comme on a pu le constater plus d'une fois, irréfutable. La campagne référendaire en France en est une illustration flagrante. Voici un échantillon de cooccurrences qui font en sorte 
qu'aujourd'hui dans le discours d'un représentant de l'UMP', il n'y a aucune occurrence de ce qualificatif pendant qu'il est très présent dans le discours de Ségolène Royal. Elle en fait une sorte de blâme de son adversaire.

Les cooccurrences récurrentes du libéral: trop libéral, stigmatiser comme libéral, un acte profondément libéral, la politique des autorités taxée de libérale, frénésie libérale, un verrouillage libéral, néo-libéral, ultra-libéral, ultra ultralibéral, anti-libéral, le fléau néo-libéral.

Voici les occurrences de libéral dans le discours de Ségolène Royal :

«Ma conviction est à l'inverse de cette idéologie insidieuse : c'est en restant au service des valeurs de justice et de progrès que nous pourrons résister le plus efficacement aux vents mauvais d'un libéralisme sans foi ni loi et saisir ensemble les opportunités d'une mondialisation qui porte dans ses flancs le pire comme le meilleur, selon ce que nous saurons en faire». (S.R. 29/09/06)

«Quels qu'aient été nos votes lors du référendum de l'an dernier, nous voulons une Europe plus concrète, plus protectrice, et donc moins libérale ». (S.R.11/10/06)

Prenons à présent le terme social qui s'associe plutôt aux socialistes. Nous en avons étudié les cooccurrences dans les discours des deux présidentiables :

S. R. - un ordre social juste, les protections sociales, les forces sociales, la sécurité sociale professionnelle, un pacte social, une justice sociale, une démocratie sociale, un dialogue social, un progrès social, le lien social ;

N. S. - une crise sociale, la fracture sociale, la désintégration sociale, le progrès social contre l'économie, les charges sociales.

En effet, on peut constater déjà les tentatives du côté de la droite allant dans le sens de s'accaparer le mot et à force de le plonger dans un univers négatif, de le rendre péjoratif.

\subsection{Les marqueurs discursifs des présidentiables - « image de marque »}

Aujourd'hui plus que jamais avant, comme le dit COTTERET (1991: 9), «le pouvoir appartient aux plus apparents ». Et le journalisme d'investigation s'étant transformé en journalisme d'inquisition, les journalistes s'emparent de toute parole pour en conserver les éléments les plus spectaculaires mais pas nécessairement les plus éclairants. Nous présenterons, au cours de cette partie, les marqueurs discursifs étant une sorte d'image de marque pour les deux politiques. Le fait est que nous vivons dans une époque où tout passage verbal pour qu'il laisse des traces mémorielles, se retient par des formules saillantes et fortes, celles qui prennent. Par exemple, Jacques Chirac est connu pour son omniprésent adverbe « naturellement», mais également l'adjectif «abracadabrantesque ». En effet, ce phénomène pourrait s'expliquer par au moins trois raisons, notamment, que l'on fonctionne aujourd'hui en communication par flash. L'urgence oblige. Il s'agit de trouver une formule sémantiquement forte et saillante, une sorte d'étiquette sémantique qui permet vite d'associer tel mot, syntagme ou formule à un politique concret. La cause en est également dans le fonctionnement par mot-clé des moteurs de recherche sur le réseau. Et enfin, un processus linguistique banal selon

\footnotetext{
${ }^{6}$ Tout en sachant que les libéraux font partie du groupe UMP.
} 
lequel une belle formule se lexicalise et continue à exister dans le langage standard.

Voyons à présent les marqueurs discursifs des deux politiques:

N. S. - le karcher, la racaille, les voyous, je persiste et je signe, la rupture, la discrimination positive ${ }^{7}$.

« Nous allons nettoyer la cité des 4000 au Karcher » (19/06/05)

«Oui, Madame, faites-moi confiance, on va vous débarrasser de ces racailles ! » (10/07/05)

« Ce sont des voyous, des racailles, je persiste et je signe ! (10/11/05)

Pour avoir prononcé ces quelques formules, N. Sarkozy a dû s'expliquer plus d'une fois. Bref, elles ne le quittent plus, il en a d'ailleurs fait un atout de campagne, on parle désormais de son franc-parler, de son parler-vrai car comme il l'a dit dans des formules désormais bien connues :

«Un homme libre se doit de parler librement » (10.09.05) ou

«Si le peuple vous a quittés c'est parce que vous ne parlez pas comme lui. » (10.09.05)

S. R. - le désir d'avenir, la démocratie participative, les gens sont spécialistes (experts) de ce qui les concernent - l'intelligence collective ; mon projet, il est socialiste $^{8}$, mettre de l'ordre dans le désordre.

«La seconde idée-force, c'est donner un avenir à la France, un désir d'avenir : les Français sont créatifs, ils ont du talent, les territoires sont attractifs. » (16/09/06)

«C'est pourquoi la démocratie participative est une façon de répondre à la crise démocratique». (29/09/06)

«Ca fait partie aussi de la démocratie participative, où les partenaires sociaux se saisissent de leurs problèmes, parce qu'ils sont les meilleurs experts de ce qu'ils vivent. » (17/10/06)

«Cela peut vous sembler abstrait, ce n'est pas un catalogue de mesures, mais je crois que c'est en réalité très concret car il s'agit de construire une intelligence collective des mutations en cours qui fasse place à l'expérience sensible de chacun». (25/08/06)

«Alors il s'agit de mettre fin au désordre des choses, au désordre de la précarité qui sape la valeur travail, au désordre de la baisse du pouvoir d'achat qui sape la confiance en soi, à l'anarchie financière qui sape la vraie valeur économique et au désordre de l'environnement qui met en cause l'avenir de la planète. » (17/10/06)

Pour SALAVASTRU (2004 : 59), nous vivons à l'ère de la «tyrannie du mot » car l'effet d'aujourd'hui tend à la personnalisation (CAYROL, 1986:129) de la politique. Il s'ensuit que la politique devient une scène de rivalité entre

\footnotetext{
${ }^{7}$ L'idée vient des Etats-Unis et Nicolas Sarkozy en est précurseur en France, mais son introduction date de 2003 et est donc reprise lors de cette campagne.

${ }^{8}$ C'est une allusion et riposte à un reproche formulé à Lionel Jospin lors de la campagne présidentielle en 2002, qui avait déclaré que son projet n'était pas socialiste.
} 
personnalités et leur mise en scène construite aussi sur leur identité discursive plutôt qu'entre idées politiques.

\subsection{Le squelette lexical}

Comme l'indique D. MAYAFFRE (2004:24) «l'actualité impose ses mots ». Pourtant, comme le démontrent nos analyses statistiques, il y a un vocabulaire appelé «l'architecture immuable du discours présidentiel ». C'est une sorte de tronc commun à tous les présidents qu'ils soient de gauche ou de droite et indépendamment des époques. Dans ce vocabulaire, on pouvait trouver les mots comme la France, le pays et le problème. Observons ce qu'il en est du discours des deux présidentiables. Et on constate déjà l'évolution, à savoir, même si la France et les Français font toujours partie du lexique commun et ceci avec la fréquence comparable, il y en a d'autres qui apparaissent seulement et que l'on pourrait qualifier de plus concret et affectif. Ainsi, le problème d'hier est remplacé par précarité d'aujourd'hui avec toujours une fréquence équivalente. La tendance suivante consisterait à voir apparaître toute une série de mots qui décrivent et/ou évoquent une émotion.

En effet, le choix des mots semble reposer sur quatre stratégies (Chauraudeau, 2005) :

- l'hyperbole - d'où l'inflation fréquente des chiffres et statistiques, particulièrement productif dans le discours de N. S. du fait de sa fonction au gouvernement;

- le choix des mots qui décrivent l'émotion de manière explicite - (joie, peur, colère) ;

- le choix des mots supposés déclencher l'émotion - voyou, karcher, terrorisme, précarité ;

- le caractère évocateur des énoncés qui produisent de l'émotion du fait de la situation - CPE, les 35 heures, etc.

En dehors du clivage droite/gauche, chez les deux candidats nous avons sélectionné un noyau dur commun relatif aux sujets abordés, notamment: le travail - travailler, l'emploi, le (la) politique.

$\mathrm{Si}$ on compare les analyses lexicales statistiques de ces deux candidats et les analyses effectuées sur le discours des présidents de la $\mathrm{V}^{\mathrm{e}}$ République, il en ressort quelques tendances dans l'évolution du discours politique. Premièrement, l'importance croissante accordée à la fonction phatique dans ce discours, on observe le souci d'ancrer le discours dans l'immédiat, de l'accrocher à la réalité. Alors qu'avant, c'était plutôt la distance qui régnait avec un niveau d'abstraction assez élevé, maintenant un passage s'opère vers le concret d'où de nombreux exemples, le recours au vécu, les citations et enfin les témoignages :

«Beaucoup de Français ont le sentiment de ne plus être ni écoutés ni compris. Et moi je préfère un socialisme qui regarde les choses en face, la réalité telle qu'elle est, la façon dont vivent les gens pour prendre en compte leurs aspirations, leurs besoins, leurs frustrations, leur mal être, parce que, être socialiste, c'est répondre à ces frustrations et à ce mal vivre, plutôt que de réciter des formules pour faire croire que tout ce qu'on a fait était parfait. » (S.R. 11/10/06) 
« J'ai reçu une lettre bien émouvante d'une vieille dame de 87 ans qui m'écrit de Marmande: (...) Elle a raison. Cette reconnaissance est un droit. Je me battrai pour ce droit. (N.S. 22/06/06)

Une autre tendance allant dans le même sens est de simplifier le langage politique, de le rendre plus accessible en évitant les mots renvoyant aux stratégies politiques ou à l'idéologie, très peu de mots savants ou scientifiques, peu de termes en -isme comme le socialisme, le gaullisme. Ce langage tend à s'appauvrir, à se détechniciser. (CAYROL, $1986: 73$ )

Le discours politique participe à la mise en scène du politique d'où le jeu de pronoms personnels et les différents procédés énonciatifs (CHAURAUDEAU, 2005 :134). Ils permettent à celui qui parle:

- de se mettre en scène (énonciation élocutive) - l'emploi des pronoms personnels de première personne accompagnés de verbes de modalité, d'adverbes et de qualificatifs : je veux, je crois (cf. les exemples de la p.7).

Ceci permet de créer l'ethos d'engagement, mais tout en posant l'action à réaliser dont la réalisation ne dépend pas de lui (ou ne dépend pas que de lui). En d'autres termes, il se donne une position de «pouvoir le faire », puisque c'est lui qui fait l'offre, mais le résultat dépend de l'acceptation de l'interlocuteur ;

- d'impliquer son interlocuteur dans sa prise de parole (énonciation allocutive) de par l'emploi des pronoms personnels de deuxième personne également accompagnés de verbes de modalité et de qualificatifs et de diverses dénominations, qui révèlent à la fois l'implication de l'interlocuteur, la place que lui assigne le locuteur, et la relation qui s'instaure entre eux :

«Vous voulez être libres ! Vous avez raison. Sachez que cette liberté se mérite. L'ignorant ne peut pas être libre. (...)»

«Vous voulez sauver la planète et vous avez raison». (N.S. 03/09/06)

«Vous êtes la génération de la communication et des marques. »

«Vous êtes la génération d'internet, du portable, du rap et de la techno. » (N.S. 03/09/06)

«Vous êtes notre avenir et avec vous, nous définirons une nouvelle donne.

C'est à cela que je vous invite. » (S.R 05/10/06)

- et enfin de présenter ce qui est dit comme si personne n'était concerné (énonciation délocutive) :

« Mais cela, les Français n'en veulent pas et ils ont bien raison !» (S.R. 01/10/06)

»Les Français veulent y voir clair sur ce qui bouge et sur ce qui perdure. Ils veulent savoir au nom de quelles valeurs il est possible d'agir. » (S.R. 25/08/06)

Sémantiquement parlant, tout énoncé se classe (SALAVASTRU, 2004 :42) selon qu'il sert à désigner, les désignatifs - pour constater la situation telle qu'elle est :

«Et moi je préfère un socialisme qui regarde les choses en face, la réalité telle qu' elle est, la façon dont vivent les gens pour prendre en compte leurs aspirations, leurs besoins, leurs frustrations, leur mal être, parce que, être socialiste, c'est répondre à ces frustrations et à ce mal vivre, plutôt que de réciter des formules pour faire croire que tout ce qu'on a fait était parfait.» (S.R. 17/10/06); 
ensuite l'énoncé prescriptif dont le rôle est d'indiquer la façon de se comporter face à cette situation, souvent avec une allure polémique :

«Vous ne la sauverez pas en reniant l'économie, la science et le progrès mais en les mettant au service d'un développement durable. Le développement durable ce n'est pas la fin du travail, c'est l'emploi durable. Ce n'est pas la croissance zéro, c'est la croissance durable. Ce n'est pas le rejet de la technique, c'est la technologie propre.» (N.S.03/09/06)

Viennent juste après les appréciatifs qui présentent la description de la situation et les formatifs - les déterminants, les connecteurs et modalités.

\subsection{La dynamique syntaxique}

Le mode conversationnel élaboré sur les blogs ne reste pas sans impact sur le discours politique en dehors de la toile.

Syntaxiquement parlant, nous avons affaire, dans les deux cas, au discours verbal plutôt que nominal en suivant la tendance exprimée déjà par MAYAFFRE (2004 : 34) : « le discours nominal (récurrence des déterminants, noms et adjectifs) du début de la période se transforme en un discours verbal (récurrence des pronoms, des verbes et des adverbes) au tournant des années 80 ».

L'on peut diviser les verbes et les pronoms personnels (E. RAULET, 2004) en trois catégories :

- les verbes de perception servant à évaluer l'action ;

- les verbes d'action qui servent à décrire l'action en rendant ainsi le discours plus actif et moins distant ;

- les verbes d'orientation (verbes modaux) qui décrivent l'attitude de l'énonciateur à l'égard de son propre énoncé, son engagement par rapport à son discours ; il s'agit des verbes : vouloir, pouvoir, devoir, croire et savoir. Parfois on y classe également le verbe falloir. Puisque l'époque est à la mise en scène de celui qui parle, on aura donc une fréquence croissante de verbes de cette catégorie :

« Je veux m'adresser à celui qui a quitté l'école sans aucune formation et qui n'en peut plus de dépendre de ses parents

Je veux m'adresser à l'habitant de la commune rurale qui

Je veux m'adresser au petit commerçant qui

Je veux m'adresser à cette mère de famille ». (N.S. 22/05/06)

«Je veux faire de la France un pays exemplaire » (S.R. 11/10/06)

« Je crois, moi, que la gauche est la seule capable de remettre l'éducation au cour de tout, de remettre l'école d'aplomb, de remettre de la culture partout, sur tout le territoire, de donner à chaque enfant le droit à la culture et à une ambition scolaire ainsi qu'à la récompense de l'effort. » (S.R. 29/09/06)

En disant je veux, N. Sarkozy ou S. Royal expriment une action à réaliser. Le fait qu'ils sont dans une situation de manque mais révèlent en même temps qu'ils n'ont pas le pouvoir de combler ce manque et qu'ils ont besoin d'avoir recours à un tiers pour accomplir l'action. (CHARAUDEAU, 1992 : 610). 
Et on voit à travers ces exemples justement que ces verbes d'orientation s'inscrivent dans un souci de cohérence intradiscursive, d'où la reprise de la formule qui donne par ailleurs de l'expressivité. Cette mise en relief permet encore plus d'insister sur ce rapport entre celui qui parle et le dit. Et cet autocentrage fait partie de la mise en scène du politique.

Ceci explicite l'importance donnée au public, l'engagement du «je » par rapport à « vous » que le politique prétend impliquer dans le projet. La présence également du pronom personnel non exclusif de «nous» l'expriment encore mieux,

«Oui, il est possible de construire la France dont vous rêvez et je veux la construire avec vous. Nous la construirons ensemble. Si nous sommes ensemble, unis, déterminés, tout sera possible. Ou bien nous ne changeons rien et nous continuons, ou bien nous changeons tout dans notre façon de concevoir la politique et nous construisons réellement une France nouvelle. » (N.S. 03/09.06)

«Nous devons remettre la France en mouvement, dans une cohérence retrouvée. »

«Seule, je ne peux rien, mais si nous sommes de plus en plus nombreux, alors tout devient possible ! » (S.R. 16/09/06)

On remarque la prédominance du «nous » qui est passé de ce héros collectif renvoyant à la famille politique, aux militants ou au gouvernement. Il est plus le plus souvent relatif aujourd'hui à nous englobant tout et un chacun. Quant à l'emploi temporel, ce sont le présent de l'indicatif avec les valeurs de présent actuel et générique et le passé composé exprimant le présent accompli, qui priment ce qui permet d'ancrer le discours dans l'immédiat du moment.

De ce qui précède résulte que la syntaxe relève surtout de l'écrit oralisé et tend vers le français parlé.

\section{Du politiquement correct au politiquement direct}

Le public d'un blog s'est habitué à vivre un nouveau mode de relations avec le politique, et il l'exige aussi en dehors de la toile. Ainsi, le politique a dû abandonner le ton institutionnel qui fait un effet de sérieux, voire trop sérieux et officiel au profit d'un ton plus personnel, direct et ouvert. Il s'agit d'établir la confiance et d'instaurer une relation qualifiée par R. LEFEBVRE (2005:46) comme «plus transparente, plus directe et plus exclusive ».

Le fait est que le blog en tant que nouveau média a rapproché la politique et les citoyens en vue de débattre et discuter avant toute prise de décision comme l'a démontré l'exemple de la campagne référendaire portant sur le Traité de Constitution. On pourrait même dire que le politiquement correct devient aujourd'hui le politiquement direct. En d'autres termes, le politique ne se soucie plus tellement de voir si tel mot ou formule peut se dire mais regarde plutôt si le lexique et la syntaxe qu'il emploie, atteignent leur cible.

«Pour redonner de la considération à la politique il faut commencer par dire les choses telles qu'elles sont sans se demander de façon incessante si cela sera ou non populaire. » (N.S. 24/06/05) 
En voici un échantillon d'exemples prouvant ce souci d'un côté de paraître proche, de l'autre de rapprocher la politique plus globalement ce que l'on appelle aujourd'hui la transparence. En effet, la transparence est un des termes clé, très à la mode, semble être un idéal de communication en politique, en économie ou encore dans bien d'autres domaines. Il s'agirait pourtant, de regarder de près ce que l'on en fait. Plusieurs niveaux de transparence sont ici à envisager. Premièrement, l'évoquer systématiquement dans son discours est aujourd'hui un passage obligé de tout homme politique, l'objectif premier étant de faire un effet de transparence :

« Vous voulez la vérité ! Cela tombe bien : je refuse le mensonge ! (N.S. $13 / 11 / 06)$

«Je veux tout mettre sur la table sans tabou. » (N.S. 06/07/06)

«Trop longtemps, on a expliqué aux Français que l'économie c'était compliqué, que c'était une affaire de spécialistes ou de chiffres. » (S.R. 17/10/06)

«La première tâche de la gauche, c'est de proposer une lecture cohérente et partageable de cette « grande transformation » qui ne se borne pas aux métamorphoses du marché (...) » (S.R 25/08/06)

Ensuite, ce politique veut souvent faire preuve de transparence, mais c'est difficilement mesurable. On pourrait, à titre d'exemple, évoquer ici l'intervention critique de Ségolène Royal sur la politique des 35 heures du gouvernement Jospin, ce qui a été, par beaucoup reconnue comme relavant de la transparence:

«Les 35 heures ont été un formidable progrès social pour la plupart des salariés ; mais pour une minorité de salariés, elles ont constitué une régression sociale parce qu'elles ont été imposées dans certaines entreprises $(. .) » ..(17 / 10 / 06)$

Ou alors les actions menées par N. Sarkozy en tant que Ministre de l'Intérieur, relevant de cette discrimination positive sont également qualifiées de transparentes.

«C'est une lâcheté qui finit par devenir une complicité, que celle qui consiste à excuser l'inexcusable. Je n'ai pas l'intention de me taire devant des comportements inacceptables. » (N.S. 03/09/06)

« Si elle ne les écoute pas c'est parce qu'elle ne les comprend pas. » (N.S. 03/09/06)

La transparence présuppose également une dimension pédagogique, il s'agira d'expliquer, rendre le discours plus clair et moins abstrait:

«Beaucoup de Français ont le sentiment de ne plus être ni écoutés ni compris. La rénovation des méthodes, l'exigence d'écoute et de participation, les réformes précises et crédibles, les réponses apportées aux inquiétudes des catégories populaires détermineront non seulement le résultat pour gagner, mais surtout la confiance pour réussir. » (S.R. $11 / 10 / 06)$

«C'est une demande de sens et de compréhension d'un monde où les repères d'antan, à commencer par ceux relatifs au travail, semblent s'effondrer l'un après l'autre. La première tâche de la gauche, c'est de proposer une lecture cohérente et partageable de cette « grande 
transformation » qui ne se borne pas aux métamorphoses du marché $(\ldots)$ » (S.R. 25/08/06)

«Les Français veulent y voir clair sur ce qui bouge et sur ce qui perdure. Ils veulent savoir au nom de quelles valeurs il est possible d'agir. Ils attendent de ceux qui briguent ». (S.R.25/08/06)

«Trop longtemps, on a expliqué aux Français que l'économie c'était compliqué, que c'était une affaire de spécialistes ou de chiffres. » (S.R. 17/10/06)

Si par contre, par transparence on entend que les processus donnant lieu aux décisions doivent être compréhensibles, que les décisions elles-mêmes doivent être rationnelles aux yeux de tout le monde et que dans la mesure du possible, les informations sur lesquelles se basent les décisions doivent être rendues accessibles au public, la transparence est loin d'être établie. Elle est tout de même devenue, l'une des bases du fonctionnement en démocratie, c'est une condition nécessaire à la fois sur le plan de la responsabilité des politiques à l'égard des électeurs, et sur le plan de la participation de ces derniers aux affaires publiques. Ce revirement ne s'explique pas seulement par l'obligation de rendre compte; il est aussi dû au fait que l'on reconnaît maintenant que le politique en faisant preuve de transparence, aurait plus de fiabilité et de légitimité.

\section{Du dialogue des sourds au dialogue permanent}

Comme, l'effet de compétence est de plus en plus remplacé par l'effet d'écoute et d'ouverture, un autre déplacement s'opère au sein du discours politique, notamment celui d'aller vers la consultation et l'interaction. Bref, le concept ancien de la démocratie participative revient en force :

« Je vous demande de m'aider à agir si vous ne voulez pas subir » (N.S. 03/09/2006)

«Je ne suis pas venu vous proposer de construire votre avenir à votre place. Je suis venu vous proposer de construire ensemble une société où chacun recevra selon son mérite et où chacun aura sa chance.» (N.S. 03/09/2006)

«Seule, je ne peux rien, mais si nous sommes de plus en plus nombreux, alors tout devient possible !» (S.R. 16/09/06)

«Aimez la France ! Elle le mérite. Donnez-vous les moyens de la transformer si vous trouvez qu'elle n'est pas à la hauteur de l'idée que vous vous faites d'elle. Prenez vos responsabilités, mobilisez-vous, agissez pour l'élever jusqu'à l'idéal que vous souhaitez la voir incarner. » (N.S.03/09/06)

En effet, le blog et plus globalement Internet fournit cette dimension participative à ne pas négliger dans le discours politique d'aujourd'hui.

\section{En guise de conclusion}

La discussion se fait désormais entre politiques et citoyens dont découlent les conséquences pour la forme aussi bien que pour le fond des discours politiques. Et comme la communication politique et les dispositifs mis à ses services changent, 
la légitimité de l'univers politique n'étant plus fondée sur la distance, il se rapproche considérablement du citoyen, en lui confiant plus de responsabilités et offrant plus d'informations, en le mobilisant, en lui proposant un espace débat et en l'aidant dans ses choix politiques. Ce discours a aujourd'hui une vocation pédagogique et explicative, incitative et mobilisant, expressive et concrète et donc par cela même, il est devenu moins solennel, distant, officiel et officialisé, policé et modéré. Ce discours a changé de tonalité, d'un ton institutionnel et technocratique, nous allons vers un ton plus personnel et personnalisé, d'où le relâchement (comme disent certains) du style, la simplicité avec la langue simplifiée qui utilise les conjugaisons les plus élémentaires (le présent indicatif et le passé composé). «La préoccupation illocutoire est constante et la performance pragmatique semble sa raison d'être » (MAYAFFRE, 2004 :121). Les trois termes qui présupposent toute parole politique sont désormais clarté, simplicité et efficacité. Et le langage tenu, pour être efficace doit être avant tout émotionnel plutôt que rationnel. Tout politique cherche à travers son discours à parler de soi, du projet (pour S.R qui est à co-construire et chez N.S. - préétabli mais à appliquer ensemble) et de l'autre en l'occurrence des citoyens, des adversaires. A cet égard, trois exigences sont évoquées par SALAVASTRU (2004 :178); celle de rationalité, de problématicité et d'expressivité. L'ordre rationnel de la structure du discours politique renvoie aux formes de raisonnement que l'on emploie pour persuader l'interlocuteur. Par contre, l'ordre problématique met en relief les contenus de pensée véhiculés par l'intermédiaire du discours politique. Et enfin, l'influence du discours politique sur le public est déterminée par l'expressivité de sa forme discursive; le style, sa rhétorique plus ou moins imagée. La performance discursive maximale en politique s'obtient par la coopération de ces trois dimensions.

Certes, le discours politique, sous l'influence de l'avènement de dispositifs médiatiques nouveaux, subit les transformations. Comme le constate S. OUARDI (MAAREK, 2004) « les médias informatisés sont une opportunité, voire même une opportunité unique de revivifier un débat démocratique dont l'essoufflement semble ne plus porter à discussion. Une telle lecture prenant pour appui les caractéristiques techniques de l'objet qui promettent une prise de parole permise à tous, vers tous, avec une évacuation présumée des intermédiaires et un accès universel vers une information universelle et surtout transparente. » Alors que jusqu'à il y a encore très peu, on pouvait réfléchir aux circulations et aux transformations éventuelles des discours d'un support à l'autre, aujourd'hui, il faut parler de la multicanalité du même discours. En effet, la cohérence oblige, le discours des autres médias sont transférés sur internet et ceux d'internet alimentent à leur tour les autres médias.

La toile aspire à plus de dialogue, plus de démocratie et de participation. De nouveaux espaces de sociabilité politique émergent. Car c'est bien la question des espaces de débat en démocratie que l'arrivée des médias informatisés en communication électorale vient raviver.

Nous avons tenté de le prouver, les deux candidats en question marquent chacun à sa façon et avec leurs spécificités, le tournant dans le discours politique qui ne fait que s'adapter aux circonstances, aux attentes du public, aux dispositifs et aux idées politiques en mouvement. Cette évolution les concernent en dehors du clivage droite/gauche. Les dérives existent déjà, notamment une surinterprétation 
du terme transparence qui a été, aux besoins des journalistes, reconduite sur non seulement les procédés en politiques mais sur les hommes et femmes politiques d'où l'apparition du phénomène de «politique people » ou de la «peopolisation » de la politique.

On pourrait s'interroger sur l'adéquation du discours à la cible visée et à laquelle il s'adresse mais, le résultat du scrutin nous en dira déjà beaucoup, surtout que « la cible du discours d'aujourd'hui, surtout celle du discours politique, n'est pas un groupe restreint, comme dans la rhétorique ancienne (tribunaux, parlement, sénat, amphithéâtre), mais, si possible, le monde entier, le peuple d'un pays en tout cas. » (SALAVASTRU, 2004 : 198).

Le discours politique est loin d'être statique, et la preuve en est dans le fait que l'évolution s'opère devant nos yeux et au sein des partis, ainsi le discours de S. Royal est en rupture par rapport à celui de D. Strauss-Kahn ou de L. Fabius tout comme le discours de Nicolas Sarkozy s'oppose dans sa forme et dans son contenu à celui de Michèle Alliot-Marie ou de Dominique de Villepin.

\section{BIBLIOGRAPHIE}

CAYROL R. (1986), La nouvelle communication politique, Paris, Larousse.

Chauradeau P. (1992), Grammaire du sens et de l'expression, Paris, Hachette.

CHAURADEAU P. (2005), Le discours politique ; Les masques du pouvoir, Paris, Vuibert.

COTTERET J.-M. (1991), Gouverner c'est paraître, réflexions sur la communication politique, Paris, PUF.

GARAPAN A. (1996), Le gardien de promesses, Justice et démocratie, Paris, Editions Odile Jacob.

Kerbrat-Orecchioni C., Mouillaud M. (1984), Le discours politique, Presses Universitaires de Lyon.

LEFEBVRE R. (2005), Rapprocher l'élu et le citoyen. La «proximité » dans le débat sur la limitation du cumul des mandats (1998-2000), in Mots. Les langages du politique, n. 77 mars 2005, pp. 41- 57, Lyon, ENS Editions.

MAYAFFRE D. (2002), Discours politique, genres et individualisation sociolinguistique. In Actes des JADT - Journées Internationales d'analyse statistique des données textuelles, p. 517-529.

MAYAFFre D. (2004), Paroles de président, Paris, Honoré Champion Editeur.

OUARDI S. (2004), Internet dans la campagne présidentielle 2002 : un pas de plus vers l'appriopriation des médias informatisées in MAAREK PH.-J, La communication politique française après le tournant de 2002, Paris, l'Harmattan, p. 109-126.

PhaAdT L. (2006), Sarkozy décrypté de A à Z, Grainville, City Editions.

RAULET E. (2004), L'analyse lexicale des discours de candidature, in MAAREK PH.-J, La communication politique française après le tournant de 2002, Paris, l'Harmattan, p. 59-78.

SAlAVASTRU C. (2004), Rhétorique et politique; Le pouvoir du discours et le discours du pouvoir, Paris, L'Harmattan. 
<http://www.cavi.univ-paris3.fr/lexicometrica/jadt/jadt2004/pdf/JADT_075.pdf > $<\mathrm{http}: / /$ www.desirsdavenir.org/>

$<$ http://blog-ump.typepad.fr/blog/>

$\langle$ http://www.sarkozynicolas.com/index.php>

$<$ http://sarkozyblog.free.fr/>

\begin{abstract}
At the hour of the Net even the policies convert with the blog which thus became a new instrument of expression, of political consultation but especially of propaganda. We would attend with the advent of new media, the birth of a new form of democracy and finally the appearance of the policy with more human face which with a different writing passes from politically correct to politically direct, of an institutional speech to the more personal tone. Indeed, the political speech changes considerably, and this more and more quickly as well in its form in the content. The object here is to determine the dynamics in which this form began of expression.
\end{abstract}

